\title{
BMJ Open Toxoplasma gondii seroprevalence in the Portuguese population: comparison of three cross-sectional studies spanning three decades
}

\author{
Maria João Gargaté, ${ }^{1}$ Idalina Ferreira, ${ }^{1}$ Anabela Vilares, ${ }^{1}$ Susana Martins, ${ }^{1}$ \\ Carlos Cardoso, ${ }^{2}$ Susana Silva, ${ }^{3}$ Baltazar Nunes, ${ }^{3}$ João Paulo Gomes ${ }^{4}$
}

To cite: Gargaté MJ, Ferreira I, Vilares A, et al. Toxoplasma gondii seroprevalence in the Portuguese population: comparison of three crosssectional studies spanning three decades. BMJ Open 2016:6:e011648. doi:10.1136/bmjopen-2016011648

- Prepublication history for this paper is available online. To view these files please visit the journal online (http://dx.doi.org/10.1136/ bmjopen-2016-011648).

Received 23 February 2016 Revised 8 June 2016 Accepted 9 June 2016

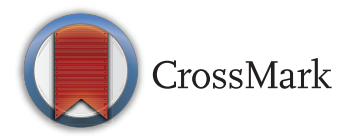

${ }^{1}$ National Reference

Laboratory of Parasitic and Fungal Infections, National Institute of Health, Lisbon, Portugal

${ }^{2}$ Clinical laboratory Dr. Joaquim Chaves, Lisbon, Portugal

${ }^{3}$ Department of

Epidemiology, National Institute of Health, Lisbon, Portugal

${ }^{4}$ Department of Infectious Diseases, National Institute of Health, Lisbon, Portugal

Correspondence to Dr Maria João Gargate; m.joao.gargate@insa.minsaude.pt

\section{ABSTRACT \\ Background: Toxoplasma gondii is an obligate intracellular protozoan infecting up to one-third of the world's population, constituting a life threat if transmitted from mother to child during pregnancy. In Portugal, there is a lack of knowledge of the current epidemiological situation, as the unique toxoplasmosis National Serological Survey was performed in 1979/ 1980.}

Methods: We studied the seroprevalence trends in the Portuguese general population over the past 3 decades, by assessing chronological spread crosssectional studies, with special focus on women of childbearing age, by age group, region and gender.

Results: The T. gondii overall seroprevalence decreased from $47 \%$ in $1979 / 1980$ to $22 \%(95 \% \mathrm{Cl}$ $20 \%$ to $24 \%$ ) in 2013 . Generally, we observed that the prevalence of $T$. gondii IgG increased significantly with age and it decreased over time, both in the general population and in the childbearing women $(18 \%$ prevalence in 2013).

Conclusions: The scenario observed for the latter indicates that more than $80 \%$ of childbearing women are susceptible to primary infection yielding a risk of congenital toxoplasmosis and respective sequelae. Since there is no vaccine to prevent human toxoplasmosis, the improvement of primary prevention constitutes a major tool to avoid infection in such susceptible groups.

\section{INTRODUCTION}

Toxoplasma gondii is an obligate intracellular protozoan parasite belonging to the phylum apicomplexa that infects most mammals worldwide. It undergoes a sexual cycle in the intestinal epithelium of the definitive host, members of the cat family, where it turns into oocysts, which are subsequently shed in the environment. It also undergoes an asexual cycle in intermediate hosts, such as birds, rodents and other mammals including human beings. ${ }^{1}$ Oocysts are extremely stable

\section{Strengths and limitations of this study}

- The results of the present study fill an important gap in the Toxoplasma gondii seroprevalence in the Portuguese population as no data were available for the past 30 years

- This study indicates that more than $80 \%$ of the Portuguese childbearing women are susceptible to primary infection, yielding a risk of congenital toxoplasmosis and respective sequelae.

- Different serological methods were used in the three cross-sectional studies, with possible sensitivity and/or specificity dissimilarities, which could not be overcome due to the chronological distance between them.

- The study of risk factors could not be performed due to the lack of more complete personal, socioeconomic and demographic information.

in the environment, and are transmitted to other hosts through inadvertent ingestion. Humans acquire T. gondii through ingestion of tissue cysts in the undercooked meat of intermediate hosts, mainly pork and lamb, or by the ingestion of water or food contaminated by faeces containing oocysts from the definitive host, ${ }^{2}$ and rarely through transplantation of an infected organ. ${ }^{3}$ While toxoplasmosis is often benign in immunocompetent individuals (revealing no symptoms or may experience swollen lymph nodes), it induces major complications in immunocompromised individuals and during pregnancy, constituting a life-threatening disease. Congenital toxoplasmosis (transmission to the fetus when a pregnant woman acquires $T$. gondii infection for the first time during pregnancy) can result in abortion or lead to severe malformation of the fetus or to visual or neurological injuries in the newborn, such as hydrocephalus, cerebral calcification and/or chorioretinitis. Such patients may require prolonged, sometimes 
lifelong, therapy. Also, recent studies suggest that subtle behavioural or personality changes may occur in T. gondii-infected humans, ${ }^{4}$ and toxoplasmosis has recently been associated with neurological disorders, particularly schizophrenia, ${ }^{5}$ and bipolar disorder. ${ }^{6}$ However, evidence for causal relationships remains limited. ${ }^{7}$

T. gondii has a wide spectrum of prevalence across the globe and infects up to one-third of the world's population. ${ }^{8}$ Cultural habits in regard to cooking food are most likely the major cause of differences in the frequency of infection with $T$. gondii in many areas of the world, ranging from $<10 \%$ to almost $100 \% .^{8-11}$ Consequently, there are large differences in the incidence of congenital infection that may vary from 1:1000 live births in France, to 1:10 000 in countries with a lower seroprevalence, and in the USA, it has been estimated that 23500 infants are born each year with congenital toxoplasmosis. ${ }^{12}$ According to a recent review, ${ }^{13}$ the global status of $T$. gondii seroprevalence in pregnant women or in reproductive ages ranges from above $60 \%$ in countries such as Brazil, Gabon, Indonesia, Germany and Iran, to $<10 \%$ in the UK and Korea. The surveillance schemes of toxoplasmosis are very heterogeneous in European countries, which hinders the burden of congenital toxoplasmosis estimates comparison. ${ }^{14}$

The first National Serological Survey was conducted in continental Portugal between 1979 and 1980 and showed T. gondii overall seroprevalence of $47 \% .{ }^{15}$ Since then, no National Serological Surveys enrolling T. gondii antibodies were performed, but only small-scale studies. ${ }^{16-18}$ Thus, Portuguese health national authorities consider that there is a lack of knowledge of the current epidemiological situation of toxoplasmosis in Portugal, and the 2011 guidelines of the General Directorate of Health for toxoplasmosis screening establish the surveillance of low-risk pregnancy based on three monthly retesting of susceptible women. ${ }^{19}$ Of note, congenital toxoplasmosis is a mandatory notifiable disease in Portugal, ${ }^{20}$ and its diagnosis should be performed in our laboratory at the National Reference Laboratory of Parasitic and Fungal Infections of the Portuguese National Institute of Health. In this regard, considering our data, there are about three cases of congenital toxoplasmosis per year in Portugal (data not published).

Considering that the unique overall population study performed in Portugal dates back to 35 years ago, the aim of this study was to describe the seroprevalence trends in the Portuguese general population (with special focus on women of childbearing age) over the past three decades (1979-1980 to 2013), by age group, region and gender.

\section{METHODS}

Study design and sampling

In order to fulfil the defined objectives, three crosssectional seroprevalence studies (1979/1980, 2001/2002 and 2013) were used. All of them were based on opportunistic sampling. Nevertheless, few biases are associated with this strategy in our study, as sera belonged to individuals seeking diverse blood analysis rather than specific T. gondii evaluation, which also reflects the vast majority of the general population. Furthermore, the same methodology was applied to all three surveys, ensuring their internal validity by increasing their comparability. The starting point was the T. gondii serological data released on behalf of the First Portuguese National Serological Survey in 1979/1980. This sample enrolled 1675 individuals of both genders, which were homogeneously distributed by the following stage groups: 8 months -5 years, $6-15,16-30,31-45$ and $\geq 46$ years. Each age group included individuals from each of the 18 districts of Portugal (Portuguese islands were not included) that were representative of the population of each district. ${ }^{15}$ Then, in this study, we processed two distinct samples. The first one was composed of sera belonging to the Second Portuguese National Serological Survey 2001-2002 that aimed to estimate the prevalence of antibodies to vaccine-preventable diseases, and for this reason the determination of antibodies for toxoplasmosis was not performed at that time. This sample enrolled 3525 individuals of both genders, and covered all age groups. Similar to the first serological survey, each age group included a number of individuals representative of the population of each of the 18 districts. For the participation of individuals, a document was prepared with the objectives and benefits of the study and informed consent was obtained either from the participants themselves or from their legal representatives. ${ }^{21}$ From these sera, we used a subsample of 1657 sera, which was established to detect the difference in antibodies prevalence between the 1979/1980 and 2001/2002 Portuguese National Serological Surveys of at least $5 \%$, with a power of $80 \%$ and a significance level of $5 \%$. This subsample comprehended individuals of both genders, homogeneously distributed in five age groups: 8 months -5 years, $6-15,16-30,31-45$ and $\geq 46$ years. Each age group included individuals from each of the 18 districts (as in previous surveys, proportion within districts was ensured). It served as baseline for the establishment of the sample size of the 2013 T. gondii serological survey, which was composed of 1440 individuals of both genders, homogeneously distributed in the same five age groups as the previous survey. In each age group, for both surveys, individuals were distributed by 18 districts of Portugal (Portuguese islands were not included) proportionally to the resident population. For the 2013 survey, individuals were selected (to fulfil the above cited requisites of age, gender and geography) from the attendees of the private clinical laboratory of Dr Joaquim Chaves during the period of January to December 2013. This is the largest Portuguese laboratory comprehending more than 40 sampling collection units dispersed throughout the whole country.

No informed consent was obtained from each participant as, besides the information regarding gender and 
age, no further information was available to the laboratory. This procedure is in agreement with the Portuguese law No. 12/2005. ${ }^{22}$

\section{Serological analyses}

Sera regarding the 2001/2002 seroprevalence study were analysed for the presence of $T$. gondii-specific antibodies in 2012, whereas the analysis for the 2013 survey was performed in 2013, both at the National Reference Laboratory of Parasitic and Fungal Infections of the Portuguese National Institute of Health.

T. gondii IgG-specific antibodies were detected by using the automated methodology enzyme linked fluorescent assay-sensibility $99.65 \%$ (interval confidence (CI) $94.55 \%$ to $97.39 \%$ ), specificity $99.92 \%$ (CI $99.58 \%$ to $100 \%$ ) and cut-off $4 \leq$ titre $<8 \mathrm{IU} / \mathrm{mL}$, with the VIDAS TOXO IgGII commercial reagents (bioMérieux SA, Marcy-l'Étoile, France) according to the manufacturer's instructions. For the resolution of equivocal samples (ie, $4 \leq$ titre $<8 \mathrm{IU} / \mathrm{mL}$ ), we retested them using a manual methodology of the direct agglutination test-sensibility $96.22 \%$ (CI $94.55 \%$ to $97.39 \%$ ), specificity $98.80 \%$ (CI $96.46 \%$ to $99.60 \%$ ) and cut-off $4 \mathrm{IU} / \mathrm{mL}$, by using the TOXO-Screen DA commercial reagents (bioMérieux SA, Marcy-l'Étoile, France), according to the manufacturer's instructions. Most of the epidemiological surveys are based on the IgG titre because IgG antibody positive titres can be detected 2-3 weeks after infection, reaching a maximum titre within 2 months. It then declines to a baseline level that persists throughout the remainder of one's life. These methodologies were not available at the time the first National Epidemiological Survey was performed (1979/1980), which used an in house indirect immunofluorescence method. ${ }^{15}$

\section{Statistical analysis}

Statistical analysis consisted of the estimation of the seroprevalences among the categories of the variables sex, age groups and region. Differences between the estimated seroprevalences were analysed using the $\chi^{2}$ test, considering the significance level of $5 \%$. The stats package of $\mathrm{R}$ software (V.3.0.3) was used ( $\mathrm{R}$ Development Core Team. R: A language and environment for statistical computing. Vienna, Austria: R Foundation for Statistical Computing, 2013. ISBN 3-900051-07-0, URL. http://www.R-project.org).

\section{RESULTS}

In the 2013 serological survey $(n=1440)$, we observed an overall prevalence of T. gondii antibodies of $22 \%(95 \%$ CI $20 \%$ to $24 \%$ ), whereas in the 2001/2002 survey ( $\mathrm{n}=1657)$ the overall prevalence of T. gondii antibodies was $36 \%$ (95\% CI $34 \%$ to $39 \%$; figure $1 \mathrm{~A}$ ). The seroprevalence in the Portuguese population decreased by $11 \%$ (95\% CI $7 \%$ to $14 \%$ ) between $1979 / 1980^{14}$ and 2001/2002, $14 \%$ (95\% CI $11 \%$ to $17 \%$ ) between 2001/ 2002 and 2013, and 25\% (95\% CI 22\% to $28 \%$ ) between

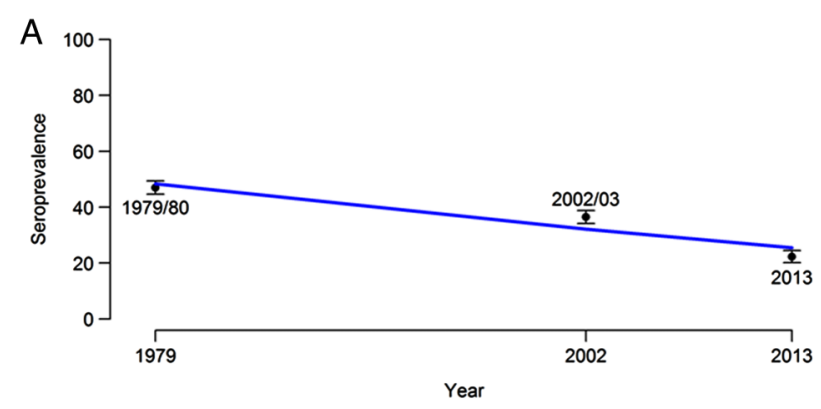

B

\begin{tabular}{|c|c|c|c|}
\hline & $\mathbf{n}$ & Seroprevalence (\%) & C1 95\% \\
\hline $1979 / 80$ & 1675 & 47 & $(45 \%-49 \%)$ \\
\hline $2002 / 03$ & 1657 & 36 & $(34 \%-39 \%)$ \\
\hline 2013 & 1440 & 22 & $(20 \%-24 \%)$ \\
\hline
\end{tabular}

Figure 1 (A) shows the evolution of Toxoplasma gondii seroprevalence in Portugal over the past three decades; (B) shows the sample sizes for the three National Serological Surveys, the precise estimated seroprevalences and respective $95 \%$ confidence interval $(\mathrm{Cl})$.

1979/1980 and 2013. The significant decreasing trend during this 34-year period is illustrated in figure 1B. Since there were no statistically significant differences between the 18 districts (data not shown), we grouped them into four regions: north, centre, Lisbon area and south. In the 2013 survey, the seroprevalences ranged from $13 \%$ (CI $95 \% 10 \%$ to $-17 \%$ ) in the north of Portugal to $33 \%$ (CI 95\% 29\% to 37\%) in the south region (table 1).

This finding is in opposition to the ones observed for the serological surveys of 1979-1980 and 2001/02, where the seroprevalence was higher in the north, centre and Lisbon area. In particular, the north region presented the highest levels in the first two surveys but the lowest in the last survey, in opposition to the south. In general, a decreasing trend was observed for the prevalence of T. gondii antibodies over the studied years in the four regions, with the exception of the south region that showed an increase of $8 \%(95 \%$ CI $2 \%$ to 19\%) between 2001/2002 and 2013 (tables 1 and 2). In particular, the decrease observed for the north region between these two surveys was statistically significant (table 2).

Regarding the association between seroprevalence and age, we observed that the prevalence of specific T. gondii IgG increased significantly with this variable for each age group (eg, for the 2013 survey, $\chi^{2}$ test for trend in proportions gives a $\mathrm{p}$ value $<0.001$ ) and it generally decreased over time (figure 2). The only exception occurred for the age group $\geq 46$ that revealed an increase from the 1979/1980 to the 2001/2002 survey. Considering solely the last two surveys (no data are available for the 1979/1980 survey, except for females between 15 and 45 years of age), the prevalence of specific $T$. gondii $\operatorname{IgG}$ decreased significantly for all age groups (except for 8 months-5 years; table 2). 
Table 1 Trends of Toxoplasma gondii seroprevalence in Portugal by region calculated with an interval confidence of $95 \%$

\begin{tabular}{lllllll}
\hline & $\mathbf{N}$ & $\begin{array}{l}\mathbf{1 9 7 9 / 1 9 8 0} \\
\text { Seroprevalence (\%) }\end{array}$ & $\mathbf{n}$ & $\begin{array}{l}\mathbf{2 0 0 1 / 2 0 0 2} \\
\text { Seroprevalence (\%) }\end{array}$ & $\begin{array}{l}\mathbf{2 0 1 3} \\
\text { n }\end{array}$ & $\begin{array}{l}\text { Seroprevalence (\%) } \\
\text { North }\end{array}$ \\
Centre & 405 & $51 \%(46 \%$ to $56 \%)$ & 426 & $45 \%(41 \%$ to $50 \%)$ & 464 & $13 \%(10 \%$ to $17 \%)$ \\
Lisbon & 590 & $47 \%(43 \%$ to $51 \%)$ & 426 & $38 \%(33 \%$ to $42 \%)$ & 344 & $29 \%(25 \%$ to $34 \%)$ \\
South & 302 & $47 \%(41 \%$ to $52 \%)$ & 630 & $33 \%(29 \%$ to $37 \%)$ & 504 & $23 \%(20 \%$ to $27 \%)$ \\
\hline
\end{tabular}

Concerning the seroprevalence distribution by gender, we observed no significant differences, either for the 2001/2002 or the 2013 survey. Both genders showed a significant decrease in prevalence between these two surveys (table 3).

\section{DISCUSSION}

In this study, we aimed to perform a cross-sectional T. gondii seroprevalence study in the Portuguese population. We acknowledge the following limitations in this serological study, namely: (1) the lack of more complete personal and socioeconomic and demographic information, hampering an evaluation involving risk factors; (2) the probable lower sensitivity and/or specificity of the serological tests used in the first national survey when compared with the ones used in the latest surveys; (3) the use of different serological methods (1979/1980 vs the two most recent surveys); and (4) the use of both fresh (1979/1980 and 2013 surveys) and frozen (2001/ 2002) sera. We believe that some of these limitations could impact some false-negative and/or false-positive results and quantitative data (ie, the determination of serological titres), but less significantly impacted the determination of the immune status of the enrolled people, which was beyond the scope of this study. Also, sensitivity and specificity of the conventional methods most likely changed little over the years, as demonstrated, for instance, by the maintenance of the gold standard (Sabin-Feldman dye test) over the past 60 years. Finally, interassay variability is very unlikely to

Table 2 Multivariate analysis adjusted for gender, age group and region

Prevalence ratios from 2002 to 2013

\begin{tabular}{ll}
\hline North & $0.37(0.28$ to 0.50$)$ \\
Centre & $0.93(0.72$ to 1.20$)$ \\
Lisbon & $0.84(0.67$ to 1.06$)$ \\
South & $1.27(0.82$ to 1.95$)$ \\
8 months-5 years & $0.60(0.13$ to 2.80$)$ \\
$6-15$ years & $0.21(0.07$ to 0.64$)$ \\
$16-30$ years & $0.52(0.35$ to 0.76$)$ \\
$31-45$ years & $0.70(0.52$ to 0.94$)$ \\
$\geq 46$ years & $0.83(0.70$ to 0.99$)$
\end{tabular}

The values represent the prevalence ratios between the 2013 and the 2001/2002 serological surveys. Data are not available for the $1979 / 1980$ survey. explain the large changes in seroprevalence observed from 1979/1980 to the 2013 survey.

The 2013 serological survey established an overall prevalence of $T$. gondii-specific IgG of $22 \%$. This value indicates a prevalence that is similar to what has been described for other Mediterranean countries, such as Spain, Italy and Greece, ${ }^{13} 23-27$ which could be associated with the similarity between these countries in terms of: (1) climate: seroprevalence may depend on the appropriate conditions for sporulation and oocysts survival in the environment, as oocysts maturation and transmission to a new host is faster at mild and wet climates, ${ }^{9}$ which are not characteristic of these countries; (2) cat contact: cats are one of the major sources of infection, as they shed a large amount of oocysts via faeces, indoors and outdoors, even after ingestion of a single bradyzoite or one tissue cyst. ${ }^{3} 28$ More, sporulated oocysts can survive for years in water, gardens, beach sands and farms, which constitute environmental features that are typical of these countries, enabling similar means of transmission due to the contact with the abundant stray and domestic cats in these environments, 328 and (3) diet: The ingestion of raw, undercooked or cured meats is the primary risk factor in Europe for acquiring toxoplasmosis, ${ }^{1}$ and these countries present the same nutritional behaviour and eating habits, namely the traditional consumption of cured meats and raw vegetables. Although the frequent cat contact and

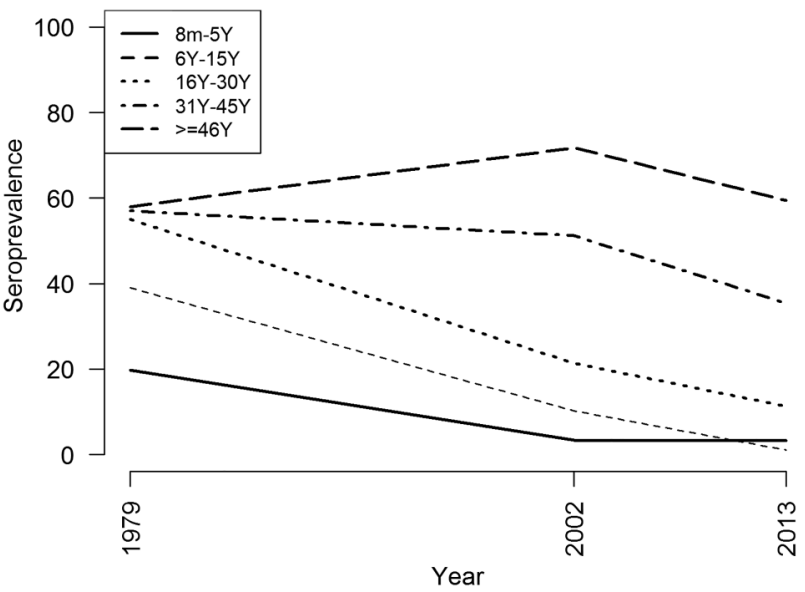

Figure 2 Comparison of Toxoplasma gondii seroprevalence trends, according to the data from three National Serological Surveys, by age groups. 
Table 3 Toxoplasma gondii seroprevalence by gender and year of analysis calculated with an interval confidence of $95 \%$

\begin{tabular}{|c|c|c|c|c|}
\hline & \multicolumn{2}{|c|}{ Male } & \multicolumn{2}{|c|}{ Female } \\
\hline & $\overline{\mathbf{N}}$ & Prevalence (\%) & $\overline{\mathbf{n}}$ & Prevalence (\%) \\
\hline 2002 & 641 & $35 \%(31 \%$ to $39 \%)$ & 1016 & $1 \%$ \\
\hline 2013 & 638 & $22 \%(19 \%$ to $25 \%)$ & 802 & $23 \%(20 \%$ to $26 \%)$ \\
\hline
\end{tabular}

Among the female population, we considered childbearing women aged $15-45$ years, and observed a significant decreasing trend in the seroprevalence in this group: $53 \%(95 \% \mathrm{Cl} 48 \%$ to $59 \%)$ in $1979-1980,35 \%(95 \% \mathrm{Cl} 32 \%$ to $38 \%$ ) in $2001 / 2002$ and $18 \%$ ( $95 \% \mathrm{Cl} 14 \%$ to $22 \%$ ) in 2013 . We also subdivided this population into five groups: $15-20,21-26,27-32,33-38$ and $39-45$ years and observed that, like in the general population, there was a significant increase of $T$. gondii seroprevalence with age (except for a unique age group within the 1979-1980 survey), and a significant decreasing trend over time (table 4).

typical diet would support higher seroprevalences than those observed, other factors, such as the climate and population education (discussed below), most likely balance this trend.

We observed a huge decrease in the $T$. gondii seroprevalence in Portugal, from 1979/1980 to the 2013 survey, as the first survey revealed a $47 \%$ rate, ${ }^{15}$ which was low compared with the values reported in France, but sharply high when compared with north European countries. Curiously, the 2013 prevalence (22\%) remains low compared with France but is now sharply lower compared with some countries from the north of Europe such as Germany, Poland, Belgium, the Netherlands and Switzerland. ${ }^{13}$ We speculate that a possible explanation for those striking differences may rely on cultural habits in regard to eating and cooking practices. The sharp decreasing trend of $T$. gondii seroprevalence observed in Portugal during the past 34 years has been also reported in many studies performed in several developed countries. ${ }^{29}$ Since the consumption of raw or undercooked meat is considered to be the major source of $T$. gondii infection, we believe that this decreasing trend may be associated with: (1) the practice of freezing meat; (2) the changes in nutritional habits that took place in Portugal and Europe during the past years related to the wide access to fast foods and pre-prepared meals, including frozen meat meals and the decreasing number of home-prepared meals; ${ }^{29}$ (3) the introduction of intensive farming techniques involving the separation of cats from livestock, coupled with the reduction of breeding cattle in backyards; ${ }^{1-29}(4)$ the release of legislation for toxoplasmosis, concerning sanitary inspection in the slaughterhouses; and (5) the improvements in health education and information by health professionals, as the lack of awareness of disease sources of transmission is a crucial factor in the risk of infection.

Regarding the distribution of $T$. gondii seroprevalence by geographic region, we observed an intriguing phenomenon as, contradicting the continuous decrease detected in the north, centre and Lisbon area, there was an $8 \%$ increase (table 1) of the seroprevalence in the south, from 2001-2002 to the 2013 survey. Also, in the 2013 survey, this region was the one with the highest T. gondii seroprevalence (33\%), which was significantly higher (about 2.5-fold) than the region showing the lowest values (north-13\%). The variation in the geoseroprevalence of this parasite may be due to the local rainy conditions and altitude. ${ }^{30}$ Nevertheless, this would explain the lower values observed for the south region in the first two surveys when compared with the other regions, but contradicts the scenario observed in the 2013 survey, as the south of Portugal presents a prominent lower altitude and is clearly drier and warmer than the remaining regions.

In most human populations, the T. gondii seroprevalence increases with age, indicating that infection is acquired throughout life. ${ }^{1}$ Our study was no exception and showed a higher seroprevalence in older age groups (for all geographical regions), most likely due to their longer exposure to the risk factors.

Concerning childbearing women, we observed a significant decreasing trend in the T. gondii seroprevalence over time (table 4). This observation is corroborated by other studies, which show that the prevalence of T. gondii infection in women of childbearing ages has decreased over the past 30 years, ${ }^{31}$ and consequently, more women are now susceptible to the infection. Besides the factors stated above that most likely justify the seroprevalence decrease in the general population, the major factor associated with the decline observed for this target group most likely relies on the promotion of educational programmes yielding an improvement of the primary prevention. The currently observed seroprevalence of $18 \%$ indicates that about $80 \%$ of Portuguese women are not immune against the T. gondii infection. Thus, the majority of potential pregnant women are susceptible to primary infection and the

Table 4 Toxoplasma gondii seroprevalence in childbearing women by age group calculated with an interval confidence of $95 \%$

\begin{tabular}{llll}
\hline & 1979 & $\mathbf{2 0 0 2}$ & $\mathbf{2 0 1 3}$ \\
\hline \hline $15-20 "$ & $51 \%(41 \%$ to $60 \%)$ & $19 \%(14 \%$ to $25 \%)$ & $4 \%(2 \%$ to $10 \%)$ \\
"21-26" & $56 \%(44 \%$ to $67 \%)$ & $23 \%(18 \%$ to $30 \%)$ & $10 \%(4 \%$ to $19 \%)$ \\
"27-32" & $46 \%(35 \%$ to $58 \%)$ & $34 \%(28 \%$ to $41 \%)$ & $17 \%(10 \%$ to $28 \%)$ \\
"33-38" & $60 \%(45 \%$ to $72 \%)$ & $48 \%(40 \%$ to $56 \%)$ & $32 \%(20 \%$ to $49 \%)$ \\
"39-45" & $60 \%(47 \%$ to $72 \%)$ & $62 \%(53 \%$ to $69 \%)$ & $50 \%(37 \%$ to $63 \%)$ \\
\hline
\end{tabular}


risk of congenital toxoplasmosis is high, a scenario that mirrors the one observed for several cities in Spain. ${ }^{32}$ The low prevalence observed among women of childbearing age should not be neglected. In fact, a recent study from the $\mathrm{WHO},{ }^{33}$ which estimated the global burden of congenital toxoplasmosis by using a previously described prediction model, ${ }^{34}$ alerts that the global burden of the disease is considerably higher than that suggested by the congenital toxoplasmosis data. The incidence of infection is dependent on the general seroprevalence, determining the population susceptibility and the frequency of risk factors for toxoplasmosis acquisition. Therefore, seroprevalence should be considered the indicator to establish screening policies. Regarding this issue, several countries have no surveillance of the infection, whereas others focus solely on severe cases and few have surveillance targeted at congenital toxoplasmosis. ${ }^{35}$ In Portugal, there is a surveillance system, which includes both the screening of pregnant women with follow-up during pregnancy of those who are not immune, in order to detect seroconversion, and the mandatory laboratory notification of congenital toxoplasmosis cases detected during this process. ${ }^{19}$

This study reinforces that prenatal screening for toxoplasmosis is necessary due to the high rate of seronegative women exposed to infection and the probability of a high number of primary infections in the childbearing period. Targeted information should be crucially provided to childbearing and pregnant women by the health professionals regarding consumption of uncooked or cured meat, raw vegetables, contaminated drinking water and contact with cats. With one exception (Toxovax for sheep), there is no approved vaccine to prevent human or animal toxoplasmosis; therefore, primary prevention is the major tool to prevent the infection in the general population, mainly in high-risk individuals, such as immunocompromised, pregnant and childbearing women. Besides these primary prevention measures, there are several actions that will determine the prevention and control of T. gondii infection, including the governmental inspection of slaughterhouses and food production industries, the improvement of hygienic standards of abattoirs, the promotion of public educational schemes and the establishment of serological screening programmes.

Contributors MJG, BN and JPG developed the study design and were responsible for data management. BN and SS performed the statistical analysis. MJG, IF, AV and SM performed the experimental assays. CC provided the sera samples. MJG and JPG wrote the manuscript.

Funding The Portuguese National Institute of Health funded this study. Competing interests None declared.

Ethics approval This study was approved by the ethical committee of the Portuguese National Institute of Health.

Provenance and peer review Not commissioned; externally peer reviewed.

Data sharing statement No additional data are available.

Open Access This is an Open Access article distributed in accordance with the Creative Commons Attribution Non Commercial (CC BY-NC 4.0) license, which permits others to distribute, remix, adapt, build upon this work non- commercially, and license their derivative works on different terms, provided the original work is properly cited and the use is non-commercial. See: http:// creativecommons.org/licenses/by-nc/4.0/

\section{REFERENCES}

1. Holliman RE. Toxoplasmosis. In: Cook GC, Zumla Al, eds. Manson's tropical diseases. Philadelphia: WB. Saunders, 2003:1365-71.

2. Cook AJ, Gilbert RE, Buffolano W, et al. Sources of toxoplasma infection in pregnant women: European multicentre case-control study. BMJ 2000;321:142-7.

3. Hill D, Dubey JP. Toxoplasma gondii: transmission, diagnosis and prevention. Clin Microbiol Infect 2002;8:634-40.

4. Flegr J. Influence of latent Toxoplasma infection on human personality, physiology and morphology: pros and cons of the Toxoplasma-human model in studying the manipulation hypothesis $J$ Exp Biol 2013;216:127-33.

5. Holub D, Flegr J, Dragomirecká E, et al. Differences in onset of disease and severity of psychopathology between toxoplasmosis-related and toxoplasmosis-unrelated schizophrenia. Acta Psychiatr Scand 2013;127:227-38.

6. Tedla Y, Shibre T, Ali O, et al. Serum antibodies to Toxoplasma gondii and Herpesvidae family viruses in individuals with schizophrenia and bipolar disorder: a case-control study. Ethiop Med J 2011;49:211-20.

7. Webster JP, Kaushik M, Bristow GC, et al. Toxoplasma gondii infection, from predation to schizophrenia: can animal behaviour help us understand human behaviour? J Exp Biol 2013;216:99-112.

8. Tenter AM, Heckeroth AR, Weiss LM. Toxoplasma gondii: from animals to humans. Int $J$ Parasitol 2000;30:1217-58.

9. Tassi P. Toxoplasma gondii infection in horses. A review. Parassitologia 2007;49:7-15.

10. Gibson CL, Coleman N. The prevalence of Toxoplasma antibodies in Guatemala and Costa Rica. Am J Trop Med Hyg 1958;7:334-8.

11. Feldman HA. Epidemiology of toxoplasma infections. Epidemiol Rev 1982;4:204-13.

12. Thulliez $P$, Remington JS. Congenital diseases learning guide. Abbott Diagnostics Europe 2013:32-53. <QC: Please check with the $\mathrm{PE}$ as this reference should be set as book since [Abbott Diagnostics Europe] is publisher not journal title. Hope I am right.>

13. Pappas G, Roussos N, Falagas ME. Toxoplasmosis snapshots: global status of Toxoplasma gondii seroprevalence and implications for pregnancy and congenital toxoplasmosis. Int $J$ Parasitol 2009;39:1385-94.

14. European Centre for Disease Prevention and Control. Annual Epidemiological Report 2013. Reporting on 2011 surveillance data and 2012 epidemic intelligence data. Stockholm: ECDC, 2013.

15. Ângelo $\mathrm{MH}$. Inquérito Serológico Nacional, Portugal continental, 1979/80 Prevalência dos anticorpos antitoxoplasmose. Arq Ins Nac Saúde 1983;8:105-9.

16. Ângelo $\mathrm{MH}$. Legal dispositions and preventive strategies in congenital toxoplasmosis in Portugal. Arch Pédiatr 2003;10:25-6.

17. Machado I. Conhecimento e prevenção da toxoplasmose na grávida - contribuição para o estudo da toxoplasmose em Portugal [dissertation]. Oporto, Portugal: University of Oporto, 2005:130 pp.

18. Lopes AP, Dubey JP, Moutinho O, et al. Seroepidemiology of Toxoplasma gondii infection in women from the North of Portugal in their childbearing years. Epidemiol Infect 2012;140:872-7.

19. Norma 37/2011, 30/09/2011 Direção Geral da Saúde. Portuguese.

20. Diário da República, 2. a série - N. ${ }^{\circ} 82-29$ de abril de 2014 Direção-Geral da Saúde Despacho n. ${ }^{\circ}$ 5681-A/2014. Portuguese.

21. Freitas MG, Paixão MT, eds. Avaliação do Programa Nacional de Vacinação: $2^{\circ}$ Inquérito Serológico Nacional Portugal Continental 2001-2002. Lisboa: Direcção-Geral da Saúde, 2004.

22. Diário da República $-1^{\text {a }}$ série- A N. ${ }^{\circ} 18-26$ de Janeiro de 2005 lei No. 12/2005 26/01/2005 pontos 6 e 9. Portuguese.

23. Ory Manchón F. Seroepidemiological surveys of non vaccine-preventable diseases and their interest in public health. Rev Esp Salud Publica 2009;83:645-57.

24. Gutiérrez-Zufiaurre N. Seroprevalence of antibodies against Treponema pallidum, Toxoplasma gondii, rubella virus, hepatitis B and Cvirus, and HIV in pregnant women. Enferm Infecc Microbiol Clin 2004;22:512-16.

25. Mosti M, Pinto B, Giromella A, et al. A 4-year evaluation of toxoplasmosis seroprevalence in the general population and in women of reproductive age in central Italy. Epidemiol Infect 2013;141:2192-5.

26. Pinto B, Castagna B, Mattei R, et al. Seroprevalence for toxoplasmosis in individuals living in north west Tuscany: access to Toxo-test in central Italy. Eur J Clin Microbiol Infect Dis 2012;31:1151-6. 
27. Diza E, Frantzidou F, Souliou E, et al. Seroprevalence of Toxoplasma gondii in northern Greece during the last 20 years. Clin Microbiol Infect 2005;11:719-23.

28. Dabritz HA, Conrad PA. Cats and Toxoplasma: implications for public health. Zoonoses Public Health 2010;57:34-52.

29. Nowakowska D, Stray-Pedersen B, Spiewak E, et al. P Prevalence and estimated incidence of Toxoplasma among pregnant women in Poland: a decreasing trend in the younger population. Clin Microbiol Infect 2006;12:913-17.

30. Flegr J, Prandota J, Sovičková M, et al. Toxoplasmosis-a global threat. Correlation of latent toxoplasmosis with specific disease burden in a set of 88 countries. PLOS ONE 2014;9:e90203.

31. Bobic BA, Nikolic IK, Djurkovic-Djakovic O. Kinetics of Toxoplasma infection in the Balkans. Wien Klin Wochenschr. 2011;123(Suppl 1):2-6.
32. Bartolomé Alvarez J, Martínez Serrano M, Moreno Parrado L, et al. Prevalence and incidence in Albacete, Spain, of Toxoplasma gondii infection in women of childbearing age: differences between immigrant and non-immigrant (2001-2007). Rev Esp Salud Publica 2008;82:333-42.

33. Torgerson PR, Mastroiacovo P. The global burden of congenital toxoplasmosis: a systematic review. Bull World Health Organ 2013;91:501-8

34. Larsen SO, Lebech M. Models for prediction of the frequency of toxoplasmosis in pregnancy in situations of changing infection rates. Int J Epidemiol 1994;23:1309-14.

35. European Food Safety Authority Panel on Biological Hazards. Scientific opinion on Surveillance and monitoring of Toxoplasma in humans, food and animals. EFSA $J$ 2007;83:1-64. 